\title{
A modified Schrödinger-type identity: uniqueness of solutions for singular boundary value problem for the Schrödinger equation
}

Hongjun $\mathrm{He}^{1 *}$ and Zhifeng Pang ${ }^{2}$

"Correspondence:

hj.he@hotmail.com

${ }^{1}$ Department of Basic Teaching,

Zhengzhou University of Industrial

Technology, Zhengzhou, China

Full list of author information is

available at the end of the article

\begin{abstract}
This paper is devoted to modifying the Schrödinger-type identity related to singular boundary value problem in (Zhang et al. in Bound. Value Probl. 2018:135, 2018). We also present some mathematical consequences of the method, including a stability result. The main technical tools used to develop the mathematical analysis are local and global bifurcation, monotonicity techniques, fixed point theory in b-metric spaces in (Liu et al. in Bull. Aust. Math. Soc. 94(1):121-130, 2016) and the maximum principle approach with respect to the Schrödinger operator in (Fan et al. in Math. Appl. 31(1):42-48, 2018). As an application, the uniqueness of solutions for singular boundary value problem for the Schrödinger equation is proved.
\end{abstract}

Keywords: Boundary value problem; Schrödinger-type identity; Uniqueness

\section{Introduction}

In this paper we consider a singular boundary value problem with mixed boundary conditions and spatial heterogeneities given by (see [4-6])

$$
\begin{aligned}
& -\Delta f=\chi f \quad \text { in } ~ \\
& f=0 \quad \text { on }\urcorner_{1}, \\
& \left.\partial f+V(t) f=\chi \omega(t) u^{q} \quad \text { on }\right\urcorner_{2}, q>1,
\end{aligned}
$$

where:

(i) $I=(0, W) \times(0, w)$ is a bounded rectangular domain in $\mathbb{R}^{2}, I$ represents a porous medium, with Lipschitz boundary $\left.\partial \beth=\urcorner_{1} \cup\right\urcorner_{2}$ where

$$
\urcorner_{2}=(\{0\} \times[0, w]) \cup([0, W] \times\{w\}) \cup(\{W\} \times[0, w])
$$

is the part in contact with air or covered by fluid and

$$
\urcorner_{1}=[0, W] \times\{0\}
$$

is the impervious part of $\partial \beth$. Let $P=\Xi \times(0, M)$, where $M>0$;

\section{Springer}


(ii) $-\Delta$ stands for the minus Laplacian operator, $\chi$ is a function of the variable $t$ satisfying

$$
c_{1} \leq \chi(t) \leq c_{2} \quad \text { a.e. } t \in(0, W)
$$

for two positive constants $c_{1}$ and $c_{2}$ and $\omega(t)$ satisfies

$$
0 \leq \omega(t) \leq 1 \quad \text { a.e. } t \in \beth
$$

(iii) the spatial heterogeneities on the boundary come given by the potentials

$\left.V, b \in \mathcal{C}(\rceil_{2}\right)$, where $b>0$ on $\rceil_{2}$ and $V$ possesses arbitrary sign in each point $\left.x \in\right\rceil_{2}$;

(iv) $\partial f(x)$ stands for the outer normal derivative of $f$ at $t \in\rceil_{2}$.

In 2008, Polidoro and Ragusa in [7] proved a Harnack inequality for the positive solutions of ultraparabolic equations of the type $L f+V f=0$, where $L$ is a linear second order hypoelliptic operator and $V$ belongs to a class of functions of Stummel-Kato type. They also obtained the existence of a Green function and an uniqueness result for the Cauchy-Dirichlet problem. In 2016, Guariglia and Silvestrov in [8] described a wavelet expansion theory for positive definite distributions over the real line and define a fractional derivative operator for complex functions in the distribution sense. In 2017, Goubet and Hamraoui in [9] investigated both numerically and theoretically the influence of a defect on the blow-up of radial solutions to a cubic NLS equation in dimension 2. Colorado in [10] showed the existence of positive bound and ground states for a system of coupled nonlinear Schrödinger-Korteweg-de Vries equations. In 2018, Khader and Adel in [11] introduced a study of the convergence analysis and error estimation of the obtained approximation solution. The FLDE is reduced to a system of algebraic equations with the help of the properties of wavelets polynomials. Rybalko in [12] studied an initial value problem for the one-dimensional non-stationary linear Schrödinger equation with a point singular potential. Zhang and $\mathrm{Gu}$ in [13] considered a three components system of nonlinear Schrödinger equations related to the Raman amplification in a plasma. In 2019, Scapellato in [14] showed some regularity properties of solutions to the elliptic equations on Herz spaces with two variable exponents. Meng in $[15,16]$ discussed the application of the new criteria for minimally thin sets with respect to the Schrödinger operator to an approximate solution of singular Schrödinger-type boundary value problems.

The classical Randon transform (see [17]) is defined by the Cauchy principal value of the singular integral. In 2018, Zhang et al. in [1] established the Schrödinger-type identity and applied it to a Schrödinger integral equation and then gave three examples where the kernel function is a Green's function for a two-order system of Schrödinger integral equations.

In this paper, we shall use this identity to study the problem (1) with a power nonlinearity and an exponential nonlinearity both of which are singular as the solutions approaches 0 . The results from previous applications are generalized and extended. In our approach, the problem is considered as a system of coupled Schrödinger boundary value problems on two half-lines. This system can exhibit different qualitative behaviors depending on the value of the vaccination-isolation reproductive number. Following the ideas of this method, we studied the existence and the behavior of periodic solutions of a generalized model with general heterogeneous coefficients, by using a continuation theorem based on 
coincidence degree theory. Regarding existence of a solution of the problem (1) with respect to the Schrödinger operator was obtained in [2] by applying the maximum principle approach for a two-order system of Schrödinger operator in [18-22]. The regularity of the solution of the problem (1) with respect to a two-order system of Schrödinger operator was also discussed in [23], where it was proved that $\omega \in C^{0}\left([0, T] ; L^{p}(\mathrm{I})\right)$ for all $p \in[1,+\infty)$ in both classes of free boundary problem of types (see [6])

$$
\operatorname{div}(\chi(t) \nabla f+\mathcal{H}(t) \omega)-\omega_{s}
$$

and

$$
\operatorname{div}(\chi(t) \nabla f+\mathcal{H}(t) \omega)-(f+\omega)_{s}
$$

and that $f \in C^{0}\left([0, M] ; L^{p}(\beth)\right)$ for all $p \in[1,2]$ in the second class.

\section{A modified Schrödinger-type identity}

For simplicity we shall denote the solution of $(1)$ as $(f, \omega)$.

Lemma 2.1 Let

$$
\left.\left.\Sigma_{1}=\right\urcorner_{1} \times(0, M), \quad \Sigma_{2}=\right\urcorner_{2} \times(0, M), \quad \Sigma_{3}=\Sigma_{2} \cap\{\phi>0\}
$$

and

$$
\Sigma_{4}=\Sigma_{2} \cap\{\phi=0\} .
$$

(i) Let $\epsilon>0, k \geq 0$ and $\varsigma \in \mathrm{D}\left(\mathbb{R}^{2} \times(0, M)\right)$ such that $\varsigma \geq 0, \varsigma=0$ on $\Sigma_{3}$. Then

$$
\int_{P} \chi\left(t_{1}\right)\left(f_{t_{2}}+\omega\right)\left(\min \left(\frac{(f-k)^{+}}{\epsilon}, \varsigma\right)\right)_{t_{2}} d t d s=0 .
$$

(ii) Let $\varsigma=0$ on $\Sigma_{2}$. Then

$$
\int_{P} \chi\left(t_{1}\right)\left(f_{t_{2}}+\omega\right)\left(\min \left(\frac{(k-u)^{+}}{\epsilon}, \varsigma\right)-\min \left(\frac{k}{\epsilon}, \varsigma\right)\right)_{t_{2}} d t d s=0 .
$$

Proof Let $\psi$ be a measure function satisfying

$$
d\left(\operatorname{supp}(\psi), \Sigma_{2}\right)>0
$$

and

$$
\operatorname{supp}(\psi) \subset \mathbb{R}^{2} \times(0, M) .
$$

Then we know that

$$
(t, s) \mapsto \pm \psi(t, t-\kappa)
$$

vanishes on $\Sigma_{2}$ for any $\kappa \in\left(-\kappa_{0}, \kappa_{0}\right)$, where $\kappa_{0}>0$. 
So

$$
\left.\int_{P}\left[\chi\left(t_{1}\right)\left(f_{t_{2}}+\omega\right) \psi_{t_{2}}(t, s-\kappa)+(1-\omega) \psi_{s}(t, s-\kappa)\right)\right] d t d s=0
$$

which yields

$$
\int_{P} \chi\left(t_{1}\right)\left(f_{t_{2}}+\omega\right) \psi_{t_{2}}(t, s-\kappa) d t d s=\frac{\partial}{\partial \kappa}\left(\int_{P}(1-\omega(t, t+\kappa)) \psi(t, s) d t d s\right) .
$$

Equation (6) still holds for any $\psi \in L^{2}\left(0, T ; H^{1}(\beth)\right)$, where $\psi=0$ on $\Sigma_{2}$ and $\psi=0$ on $I \times\left(\left(0, \kappa_{0}\right) \cup\left(M-\kappa_{0}, M\right)\right)$.

So

$$
\varsigma \in \mathrm{D}\left(\mathbb{R}^{2} \times\left(\kappa_{0}, M-\kappa_{0}\right)\right)
$$

which shows that $\varsigma \geq 0, \varsigma=0$ on $\Sigma_{3}$.

Set

$$
\psi=\min \left(\frac{(f-k)^{+}}{\epsilon}, \varsigma\right)
$$

Then (6) also gives

$$
\begin{aligned}
& \int_{P} \chi\left(t_{1}\right)\left(f_{t_{2}}+\omega\right)\left(\min \left(\frac{(f-k)^{+}}{\epsilon}, \varsigma\right)\right)_{t_{2}}(t, s-\kappa) d t d s \\
& =\frac{\partial}{\partial \kappa}\left(\int_{P}(1-\omega(t, t+\kappa)) \min \left(\frac{(f-k)^{+}}{\epsilon}, \varsigma\right)(t, s) d t d s\right):=\mathcal{G}^{\prime}(\kappa)
\end{aligned}
$$

for any $\kappa \in\left(-\kappa_{0}, \kappa_{0}\right)$, where

$$
\mathcal{G}(\kappa)=\int_{P}(1-\omega(t, t+\kappa)) \min \left(\frac{(f-k)^{+}}{\epsilon}, \varsigma\right)(t, s) d t d s
$$

So

$$
\mathcal{G}(\kappa) \geq 0=\mathcal{G}(0)
$$

for any $\kappa \in\left(-\kappa_{0}, \kappa_{0}\right)$.

By applying (7), we know that (4) holds for $\varsigma \in \mathrm{D}\left(\mathbb{R}^{2} \times(0, M)\right)$ such that $\varsigma \geq 0, \varsigma=0$ on $\Sigma_{3}$

If we put $\varsigma=0$ on $\Sigma_{2}$ and set

$$
\psi=\min \left(\frac{(k-u)^{+}}{\epsilon}, \varsigma\right)-\min \left(\frac{k}{\epsilon}, \varsigma\right)
$$


then (6) also yields

$$
\begin{aligned}
& \int_{P} \chi\left(t_{1}\right)\left(f_{t_{2}}+\omega\right)\left(\min \left(\frac{(k-u)^{+}}{\epsilon}, \varsigma\right)-\min \left(\frac{k}{\epsilon}, \varsigma\right)\right)_{t_{2}}(t, s-\kappa) d t d s \\
& =\frac{\partial}{\partial \kappa}\left(\int_{P}(1-\omega(t, t+\kappa))\left(\min \left(\frac{(k-u)^{+}}{\epsilon}, \varsigma\right)-\min \left(\frac{k}{\epsilon}, \varsigma\right)\right)(t, s) d t d s\right) \\
& :=\mathcal{K}^{\prime}(\kappa)
\end{aligned}
$$

for any $\kappa \in\left(-\kappa_{0}, \kappa_{0}\right)$, where

$$
\mathcal{K}(\kappa)=\int_{P}(1-\omega(t, t+\kappa))\left(\min \left(\frac{(k-u)^{+}}{\epsilon}, \varsigma\right)-\min \left(\frac{k}{\epsilon}, \varsigma\right)\right)(t, s) d t d s
$$

So

$$
\mathcal{K}(\kappa) \leq 0=\mathcal{K}(0)
$$

for all $\kappa \in\left(-\kappa_{0}, \kappa_{0}\right)$.

By applying (8), we know that (5) holds for $\varsigma \in \mathrm{D}\left(\mathbb{R}^{2} \times(0, M)\right)$ such that $\varsigma \geq 0, \varsigma=0$ on $\Sigma_{2}$.

\section{Uniqueness of the solution}

In this section, we shall state and prove our main result: the solution of problem (1) is unique. Let us assume that

$$
\chi \in C^{1}([0, W]) .
$$

Now, we can state our uniqueness theorem.

Theorem 3.1 The solution of the problem (1) associated with the initial data $\omega_{0}$ is unique.

Proof Let $\left(f_{1}, \omega_{1}\right)$ and $\left(f_{2}, \omega_{2}\right)$ be two solutions of the problem (1) satisfying

$$
\omega_{1}(t, 0)=\omega_{2}(t, 0)=\omega_{0}(t)
$$

a.e. in I.

Set

$$
\nu=\left(f_{1}-f_{2}\right)^{+}
$$

and

$$
\gamma=\left(1-\omega_{2}(t, s)\right) \omega_{\left\{f_{1}>f_{2}\right\}}+\left(\left(1-\omega_{2}(t, s)\right)+\left(1-f_{2 t_{2}}(t, s)\right)\right) \omega_{\left\{f_{1}>0\right\}} .
$$

It follows that

$$
\int_{P} \iota \chi\left(t_{1}\right)\left(v_{t_{2}}+\gamma\right) \varsigma_{t_{2}} d t d s \leq 0
$$

from Lemma 2.1. 
Let

$$
\varepsilon_{0}=d(\operatorname{supp}(\varsigma), \partial \beth)
$$

and

$$
\mathcal{A}_{\varepsilon_{0}}=\left\{t \in \mathbb{R}^{2} / d(t, \partial \beth)>\varepsilon_{0}\right\} .
$$

Note that the function $\chi$ admits an extension to $\mathbb{R}$ from (9), still denoted by $\chi$, such that $\chi \in C^{1}(\mathbb{R}, \mathbb{R})($ see $[24])$.

It follows that

$$
\begin{aligned}
& \int_{\mathbb{R}^{2} \times(0, M)} \iota \chi\left(t_{1}\right)\left(v_{\varepsilon t_{2}}+\gamma_{\varepsilon}\right) \varsigma_{t_{2}} d t d s \\
& =\int_{\mathbb{R}^{2} \times(0, M)} \iota\left\{\int_{\mathbb{R}^{2}}\left(v_{t_{2}}(t-x, s)+\gamma(t-x, s)\right) \rho_{\varepsilon}(y) d y\right\} \chi\left(t_{1}\right) \varsigma_{t_{2}}\left(t_{1}, t_{2}\right) d t d s \\
& =\int_{\mathbb{R}^{2}} \rho_{\varepsilon}(y)\left\{\int_{\mathbb{R}^{2} \times(0, M)} \iota\left(v_{t_{2}}(t-x, s)+\gamma(t-x, s)\right) \chi\left(t_{1}\right) \varsigma_{t_{2}}\left(t_{1}, t_{2}\right) d t d s\right\} d y \\
& =\int_{B(0, \varepsilon)} \rho_{\varepsilon}(y)\left\{\int_{P} \iota\left(v_{z_{2}}(z, s)+\gamma(z, s)\right)\left(\chi\left(z_{1}+x_{1}\right) \varsigma(z+y)\right)_{z_{2}} d z d s\right\} d y \\
& =\int_{B(0, \varepsilon)} \rho_{\varepsilon}(y)\left\{\int_{P} \iota \chi\left(z_{1}\right)\left(v_{z_{2}}(z, s)+\gamma(z, s)\right)\left(\frac{\chi\left(z_{1}+x_{1}\right) \varsigma(z+y)}{\chi\left(z_{1}\right)}\right)_{z_{2}} d z d s\right\} d y
\end{aligned}
$$

from Fubini's theorem, where $\varepsilon \in\left(0, \frac{\varepsilon_{0}}{2}\right), \rho_{\varepsilon} \in \mathrm{D}\left(\mathbb{R}^{2}\right)$ with

$$
\operatorname{supp}\left(\rho_{\varepsilon}\right) \subset B(0, \varepsilon)
$$

and $f_{\varepsilon}=\rho_{\varepsilon} * f$.

Note that

$$
z \mapsto \frac{\chi\left(z_{1}+x_{1}\right) \varsigma(z+y)}{\chi\left(z_{1}\right)}
$$

is nonnegative for all $y \in B(0, \varepsilon)$ and belongs to $C_{0}^{1}(\beth)$.

Since (10) holds for $0 \leq \varphi \in C_{0}^{1}(\beth)$, we have

$$
\int_{\mathbb{R}^{2} \times(0, M)} \iota \chi\left(t_{1}\right)\left(v_{\varepsilon t_{2}}+\gamma_{\varepsilon}\right) \zeta_{t_{2}} d t d s \leq 0,
$$

which yields

$$
\begin{aligned}
& -\int_{\mathcal{A}_{\varepsilon_{0}} \times(0, M)} \iota \chi\left(t_{1}\right) v_{\varepsilon} \varsigma_{t_{2} t_{2}} d t d s+\int_{\mathcal{A}_{\varepsilon_{0}} \times(0, M)} \iota \chi\left(t_{1}\right) \gamma_{\varepsilon} \varsigma_{t_{2}} d t d s \leq 0, \\
& \varsigma \in \mathrm{D}(\beth), \varsigma \geq 0, \quad d(\operatorname{supp}(\varsigma), \partial \beth)=\varepsilon_{0}>0, \quad \forall \iota \in \mathrm{D}(0, M), \iota \geq 0 .
\end{aligned}
$$

Put

$$
\alpha_{\epsilon}(t)=\chi\left(t_{1}\right) \int_{0}^{M} \iota v_{\varepsilon} d s,
$$

and suppose that there exists $t_{0} \in \mathcal{A}_{\varepsilon_{0}} \cap \beth$ and $\varepsilon_{1} \in\left(0, \frac{\varepsilon_{0}}{2}\right)$ such that $\alpha_{\varepsilon_{1}}\left(t_{0}\right)>0$. 
Since $\alpha_{\varepsilon_{1}}$ is continuous and $\mathcal{A}_{\varepsilon_{0}} \cap \beth$ is an open set,

$$
\overline{B\left(t_{0}, r\right)} \subset \mathcal{A}_{\varepsilon_{0}} \cap \exists
$$

and $\alpha_{\varepsilon_{1}}(t)>0$ for all $t \in \overline{B\left(t_{0}, r\right)}$, where $r>0$.

It follows that

$$
\int_{0}^{M} \iota v_{\varepsilon_{1}} d s>0
$$

in $\overline{B\left(t_{0}, r\right)}$ from (2).

Let us consider the following Dirichlet problem associated to a linear second order partial differential equation:

$$
\begin{aligned}
& -\frac{1}{\alpha_{\varepsilon_{1}}(t)} \varsigma_{t_{1} t_{1}}-\varsigma_{t_{2} t_{2}}+\frac{\int_{0}^{M} \iota \gamma_{\epsilon_{1}} d s}{\int_{0}^{M} \iota v_{\varepsilon_{1}} d s} \varsigma_{t_{2}}=\frac{1}{\alpha_{\varepsilon_{1}}(t)} \quad \text { in } B\left(t_{0}, r\right), \\
& \varsigma=0 \quad \text { on } \partial\left(B\left(t_{0}, r\right)\right),
\end{aligned}
$$

which yields

$$
\begin{aligned}
& -\sum_{i, j=1}^{2} a_{\varepsilon_{1} i j}(t) \varsigma_{t_{i} t_{j}}+\beta_{\epsilon_{1}}(t) \varsigma_{t_{2}}=\frac{1}{\alpha_{\varepsilon_{1}}(t)} \quad \text { in } B\left(t_{0}, r\right), \\
& \varsigma=0 \quad \text { on } \partial\left(B\left(t_{0}, r\right)\right),
\end{aligned}
$$

where

$$
\begin{aligned}
& a_{\epsilon_{1} 11}(t)=\frac{1}{\alpha_{\epsilon_{1}}(t)}, \quad a_{\epsilon_{1} 12}(t)=a_{\epsilon_{1} 11}(t)=0, \quad a_{\epsilon_{122}}(t)=1, \\
& \beta_{\epsilon_{1}}(t)=\frac{\int_{0}^{M} \iota \gamma_{\epsilon_{1}} d s}{\int_{0}^{M} \iota v_{\varepsilon_{1}} d s} .
\end{aligned}
$$

Note that $\left(a_{\varepsilon_{1} i j}(t)\right)_{i j}$ is strictly elliptic in $B\left(t_{0}, r\right)$ with a positive constant

$$
\min \left(\frac{1}{\max _{t \in \overline{B\left(t_{0}, r\right)}} \alpha_{\varepsilon_{1}}(t)}, 1\right)
$$

and the coefficients $\frac{1}{\alpha_{\varepsilon_{1}}}, \beta_{\epsilon_{1}}$ are in $C^{1}\left(\overline{B\left(t_{0}, r\right)}\right)$. So, by the regularity theory (see [25] for example), the problem (12) has a unique solution $\hat{\varsigma} \in C^{2}\left(\overline{B\left(t_{0}, r\right)}\right)$.

Moreover, since the function in the right side of the first equation of (12) satisfies $\frac{1}{\alpha_{\varepsilon_{1}}}>0$ in $B\left(t_{0}, r\right)$, we have $\hat{\varsigma} \geq 0$ in $\overline{B\left(t_{0}, r\right)}$ from the maximum principle (see [26]).

So (11) yields

$$
\int_{\mathcal{A}_{\varepsilon_{0}} \times(0, M)}\left\{-\iota \chi\left(t_{1}\right) v_{\varepsilon} \hat{\zeta}_{t_{2} t_{2}}+\iota \chi\left(t_{1}\right) \gamma_{\varepsilon} \hat{\zeta}_{t_{2}}\right\} d t d s \leq 0,
$$

where $\varepsilon \in\left(0, \frac{\varepsilon_{0}}{2}\right)$. 
It follows that

$$
-\hat{\zeta}_{t_{1} t_{1}}-\alpha_{\varepsilon_{1}}(t) \hat{\zeta}_{t_{2} t_{2}}+\chi\left(t_{1}\right) \hat{\zeta}_{t_{2}} \int_{0}^{M} \iota \gamma_{\varepsilon_{1}} d s=1
$$

and

$$
\begin{aligned}
& \int_{\overline{B\left(t_{0}, r\right)} \times(0, M)}\left\{-\iota \chi\left(t_{1}\right) v_{\varepsilon_{1}} \hat{\zeta}_{t_{2} t_{2}}+\iota \chi\left(t_{1}\right) \gamma_{\varepsilon_{1}} \hat{\zeta}_{t_{2}}\right\} d t d s \\
& \quad=\int_{\overline{B\left(t_{0}, r\right)}} \hat{\zeta}_{t_{1} t_{1}} d t+\int_{\overline{B\left(t_{0}, r\right)}} d t \\
& \quad=\int_{\beth} \hat{\zeta}_{t_{1} t_{1}} d t+\int_{\overline{B\left(t_{0}, r\right)}} d t
\end{aligned}
$$

from (12), which gives that

$$
\int_{\mathcal{A}_{\varepsilon_{0}} \times(0, M)}\left\{-\iota \chi\left(t_{1}\right) v_{\varepsilon_{1}} \hat{\zeta}_{t_{2} t_{2}}+\iota \chi\left(t_{1}\right) \gamma_{\varepsilon_{1}} \hat{\zeta}_{t_{2}}\right\} d t d s=\left|\overline{B\left(t_{0}, r\right)}\right|>0 .
$$

So

$$
\alpha_{\varepsilon}(t)=\chi\left(t_{1}\right) \int_{0}^{M} \iota(t) v_{\varepsilon}(t, s) d s \leq 0,
$$

where $\varepsilon \in\left(0, \frac{\varepsilon_{0}}{2}\right)$ and $t \in \mathcal{A}_{\varepsilon_{0}} \cap$ J, which yields

$$
\int_{\left(\mathcal{A}_{\varepsilon_{0}} \cap \Xi\right) \times(0, M)} l(t) v_{\varepsilon}(t, s) d t d s \leq 0,
$$

for any $\varepsilon \in\left(0, \frac{\varepsilon_{0}}{2}\right)$.

By passing to the limit as $\varepsilon \rightarrow 0$, we obtain

$$
0 \leq \int_{\left(\mathcal{A}_{\varepsilon_{0}} \cap \boldsymbol{I}\right) \times(0, M)} l(t)\left(f_{1}-f_{2}\right)^{+}(t, s) d t d s \leq 0
$$

and

$$
\int_{P} \iota(t)\left(f_{1}-f_{2}\right)^{+}(t, s) d t d s=0 .
$$

Notice that

$$
\iota\left(f_{1}-f_{2}\right)^{+}=0 \quad \text { a.e. in } P
$$

for all $0 \leq \iota \in \mathrm{D}(0, M)$, which gives that

$$
f_{1}=f_{2}:=u \quad \text { a.e. in } P .
$$

\section{Next we prove}

$$
\omega_{1}=\omega_{2} \quad \text { a.e. in } P \text {. }
$$


Put

$$
\iota(t)= \begin{cases}2\left(\frac{t}{\sigma}\right)^{2} & \text { if } t \in\left[0, \frac{\sigma}{2}\right], \\ 1-2\left(1-\frac{t}{\sigma}\right)^{2} & \text { if } t \in\left(\frac{\sigma}{2}, \sigma\right], \\ 1 & \text { if } t \in(\sigma, s-\sigma], \\ 1-2\left(1-\frac{s-t}{\sigma}\right)^{2} & \text { if } t \in\left(s-\sigma, s-\frac{\sigma}{2}\right], \\ 2\left(\frac{s-t}{\sigma}\right)^{2} & \text { if } t \in\left(s-\frac{\sigma}{2}, s\right],\end{cases}
$$

where $\sigma>0$ and $s \in(0, M]$.

Note that $\iota \in C^{1}([0, s])$ and

$$
\iota^{\prime}(t)= \begin{cases}4 \frac{t}{\sigma^{2}} & \text { if } t \in\left[0, \frac{\sigma}{2}\right], \\ \frac{4}{\sigma}\left(1-\frac{t}{\sigma}\right) & \text { if } t \in\left(\frac{\sigma}{2}, \sigma\right], \\ 0 & \text { if } t \in(\sigma, s-\sigma], \\ -\frac{4}{\sigma}\left(1-\frac{s-t}{\sigma}\right) & \text { if } t \in\left(s-\sigma, s-\frac{\sigma}{2}\right], \\ -\frac{4}{\sigma}\left(\frac{s-t}{\sigma}\right) & \text { if } t \in\left(s-\frac{\sigma}{2}, s\right] .\end{cases}
$$

Note that $\varsigma \iota^{2} \in H^{1}(P), \varsigma \iota^{2}=0$ on $\partial \beth \times(0, M)$ and

$$
\left(\varsigma \iota^{2}\right)(t, 0)=\left(\varsigma \iota^{2}\right)(t, M)=0 \quad \text { a.e. in } \beth
$$

Choosing $\pm \varsigma \iota^{2}$ as test functions for (1) (see [27] for example), we obtain

$$
\begin{aligned}
& \int_{I_{\times}(0, s)}\left[\chi\left(t_{1}\right)\left(f_{t_{2}}+\omega_{1}\right) \varsigma_{t_{2}} \iota^{2}-2 \omega_{1} \iota^{\prime} \varsigma\right] d t d s=0, \\
& \int_{I_{\times}(0, s)}\left[\chi\left(t_{1}\right)\left(f_{t_{2}}+\omega_{2}\right) \varsigma_{t_{2}} \iota^{2}-2 \omega_{2} \iota^{\prime} \varsigma\right] d t d s=0 .
\end{aligned}
$$

It follows from (16) and (15) that

$$
\begin{aligned}
0 & =\int_{I_{\times}(0, s)} \chi\left(t_{1}\right)\left(\omega_{1}-\omega_{2}\right) \varsigma_{t_{2}} \iota^{2} d t d s-\int_{I_{\times}(0, s)} 2\left(\omega_{1}-\omega_{2}\right) \iota^{\prime} \varsigma d t d s \\
& :=\mathcal{R}_{\sigma}^{1}-\mathcal{R}_{\sigma}^{2} .
\end{aligned}
$$

By using the Lebesgue theorem to $\mathcal{R}_{\sigma}^{1}$ (see [28]), we have

$$
\lim _{\sigma \rightarrow 0} \mathcal{R}_{\sigma}^{1}=\int_{\beth \times(0, s)} \chi\left(t_{1}\right)\left(\omega_{1}-\omega_{2}\right) \varsigma_{t_{2}} d t d s .
$$

We now estimate $\mathcal{R}_{\sigma}^{2}$. We know that

$$
\begin{aligned}
\left|\mathcal{R}_{\sigma}^{2}\right| & =2\left|\int_{\beth} \int_{0}^{\sigma} \chi\left(t_{1}\right)\left(\omega_{1}-\omega_{2}\right) \iota^{\prime} s d t d s+\int_{\beth} \int_{s-\sigma}^{s} \chi\left(t_{1}\right)\left(\omega_{1}-\omega_{2}\right) \iota^{\prime} s d t d s\right| \\
& \leq C\left\{\int_{0}^{\sigma}\left(\int_{\beth}\left|\omega_{1}-\omega_{2}\right| d t\right) \iota\left|\iota^{\prime}\right| d s d s+\int_{s-\sigma}^{s}\left(\int_{\beth}\left|\omega_{1}-\omega_{2}\right| d t\right) \iota\left|\iota^{\prime}\right| d s\right\} \\
& :=C\left(\mathcal{R}_{\sigma}^{2,1}+\mathcal{R}_{\sigma}^{2,2}\right)
\end{aligned}
$$


from the definition of $\iota^{\prime}$, where

$$
C=\sup _{\left(t_{1}, t_{2}\right) \in \Xi}\left|\chi\left(t_{1}\right) \varsigma\left(t_{1}, t_{2}\right)\right|
$$

We have

$$
\omega_{1}-\omega_{2} \in C^{0}\left([0, M] ; L^{1}(\mathrm{I})\right)
$$

(see [29, Proposition 2.1]), $\iota \in C^{0}([0, s]), \iota(0)=0$ and $\iota$ is uniformly bounded independently of $\sigma$.

Notice that

$$
t \mapsto\left(\int_{\beth}\left|\omega_{1}-\omega_{2}\right| d t\right) \iota
$$

is right-continuous and vanishes at 0 .

So

$$
\lim _{\sigma \rightarrow 0} \mathcal{R}_{\sigma}^{2,1}=0
$$

Similarly,

$$
\lim _{\sigma \rightarrow 0} \mathcal{R}_{\sigma}^{2,2}=0
$$

By letting $\sigma \rightarrow 0$ in (19) and applying (20)-(21), we obtain

$$
\lim _{\sigma \rightarrow 0} \mathcal{R}_{\sigma}^{2}=0
$$

Combining (17), (18) and (22), we obtain

$$
0=\int_{0}^{s} \int_{\beth} \chi\left(t_{1}\right)\left(\omega_{1}-\omega_{2}\right) s_{t_{2}} d t d s:=F(s),
$$

where $s \in[0, M]$.

Since

$$
\int_{\beth} \chi\left(t_{1}\right)\left(\omega_{1}-\omega_{2}\right) \zeta_{2} d t
$$

is continuous on $[0, M]$ (see [30]), we deduce that

$$
F^{\prime}(s)=0
$$

for all $s \in[0, M]$ from (23).

So

$$
\int_{\beth} \chi\left(t_{1}\right)\left(\omega_{1}-\omega_{2}\right)(\cdot, s) \varsigma_{t_{2}} d t=0 \quad \forall t \in[0, M], \forall \varsigma \in \mathrm{D}(\beth),
$$


which yields

$$
\int_{\mathbb{R}^{2}} \chi\left(t_{1}\right)\left(\left(\omega_{1}-\omega_{2}\right)(\cdot, s)\right)_{\varepsilon} \varsigma_{t_{2}} d t=0
$$

If we choose

$$
\min \left(\frac{\left(\left(\omega_{1}-\omega_{2}\right)(\cdot, s)\right)_{\varepsilon}^{+}}{\sigma}, 1\right) \varsigma
$$

as the test functions in (25), then we have

$$
\begin{aligned}
0= & \int_{\mathbb{R}^{2}} \chi\left(t_{1}\right)\left(\left(\omega_{1}-\omega_{2}\right)(\cdot, s)\right)_{\varepsilon} \varsigma_{t_{2}} \min \left(\frac{\left(\left(\omega_{1}-\omega_{2}\right)(\cdot, s)\right)_{\varepsilon}^{+}}{\sigma}, 1\right) d t \\
& +\int_{\mathbb{R}^{2}} \chi\left(t_{1}\right)\left(\left(\omega_{1}-\omega_{2}\right)(\cdot, s)\right)_{\varepsilon} \min \left(\frac{\left(\left(\omega_{1}-\omega_{2}\right)(\cdot, s)\right)_{\varepsilon}^{+}}{\sigma}, 1\right)_{t_{2}} \varsigma d t \\
:= & S_{\sigma}^{1}+S_{\sigma}^{2} .
\end{aligned}
$$

By applying the Lebesgue theorem to $S_{\sigma}^{1}$, we obtain

$$
\lim _{\sigma \rightarrow 0} S_{\sigma}^{1}=\int_{\beth} \chi\left(t_{1}\right)\left(\left(\omega_{1}-\omega_{2}\right)(\cdot, s)\right)_{\varepsilon}^{+} \varsigma_{t_{2}} d t
$$

and

$$
\begin{aligned}
S_{\sigma}^{2} & =\frac{1}{2 \sigma} \int_{\mathbb{R}^{2}} \chi\left(t_{1}\right)\left\{\min \left(\left(\left(\omega_{1}-\omega_{2}\right)(\cdot, s)\right)_{\varepsilon}^{+}, \sigma\right)^{2}\right\}_{t_{2}} \varsigma d t \\
& =-\frac{1}{2 \sigma} \int_{\mathbb{R}^{2}} \chi\left(t_{1}\right) \varsigma_{t_{2}} \min \left(\left(\left(\omega_{1}-\omega_{2}\right)(\cdot, s)\right)_{\varepsilon}^{+}, \sigma\right)^{2} d t,
\end{aligned}
$$

which yield

$$
\lim _{\sigma \rightarrow 0} S_{\sigma}^{2}=0
$$

Put

$$
\left.\beth_{\varrho}:=\beth \cup\left\{x \in \mathbb{R}^{N} \backslash \beth: \operatorname{dist}(t,\urcorner_{1}\right)<\varrho\right\},
$$

and let $\left(\delta_{0}, \omega_{0}\right)$ and $\left(\delta^{\varrho}, \omega_{\varrho}\right)$ be the principal eigenvalue associated to $-\Delta$ operator in the domains $\beth$ and $\beth_{\varrho}$, respectively (see [31]).

It is obvious that

$$
\left\|\omega_{0}\right\|_{L^{\infty}(\mathrm{I})}=1, \quad\left\|\omega_{\varrho}\right\|_{L^{\infty}\left(\beth_{\varrho}\right)}=1 .
$$

By the definitions of $\omega_{0}$ and $\omega_{\varrho}$, we obtain

$$
\omega_{0}(t)=0, \quad \partial \omega_{0}(t)<0,
$$


where $\left.t \in \partial \boldsymbol{I}=\rceil_{0} \cup\right\rceil_{1}$, and

$$
\omega_{\varrho}(t)>0 \text {, }
$$

where $t \in\urcorner_{1}$.

Put

$$
\begin{aligned}
& \eta_{0}(t):=-\partial \omega_{0}(t)>0 \quad \forall t \in \partial I, \\
& \underline{\eta}_{0}:=\min _{t \in\urcorner_{1}} \eta_{0}(t)>0, \quad \bar{\eta}_{0}:=\left\|\eta_{0}(t)\right\|_{\left.L^{\infty}(\urcorner_{1}\right)}, \\
& \bar{b}:=\|\omega(t)\|_{\left.L^{\infty}(\urcorner_{1}\right)}>0, \quad \chi:=\|\chi(t)\|_{\left.L^{\infty}(\urcorner_{1}\right)} \geq 0 .
\end{aligned}
$$

Put

$$
0<\varepsilon<\frac{\underline{\eta}_{0}}{1+\chi}
$$

and

$$
0<\frac{1}{k_{0}}<\underline{\eta}_{0}-\varepsilon(1+\chi)
$$

respectively.

It is well known that

$$
\delta^{\varrho}<\delta_{0},
$$

where $\varrho>0$.

So

$$
\lim _{\varrho \downarrow 0} \delta^{\varrho}=\delta_{0}
$$

Combining (31), (35) and (36), there exists $\varrho_{0}>0$ satisfying

$$
\delta_{1}<\delta^{\varrho}<\delta_{0}
$$

where $\varrho \in\left(0, \varrho_{0}\right]$.

Thanks to the regularity of $\omega_{\varrho}$ and $\omega_{0}$, there exists $\varrho_{1} \in\left(0, \varrho_{0}\right]$ satisfying

$$
\begin{aligned}
& \partial \omega_{\varrho}(t) \leq \partial \omega_{0}(t)+\varepsilon, \\
& \omega_{\varrho}(t) \leq \omega_{0}(t)+\varepsilon=\varepsilon,
\end{aligned}
$$

where $\varrho \in\left(0, \varrho_{1}\right]$ and $\left.t \in\right\urcorner_{1}$.

Let $\left.\bar{x}_{\varrho} \in\right\urcorner_{1}$ satisfy

$$
\omega_{\varrho}\left(\bar{x}_{\varrho}\right)=\left\|\omega_{\varrho}\right\|_{\left.L^{\infty}(\urcorner_{1}\right)} .
$$


It follows that there exists $\bar{z} \in T_{1}$ satisfying

$$
\omega_{\varrho}\left(\bar{x}_{\varrho}\right)=\left\|\omega_{\varrho}\right\|_{\left.L^{\infty}(\urcorner_{1}\right)}=\eta_{0}(\bar{z}) \varrho+o(\varrho)
$$

from (39) and (40).

Put

$$
g_{\varrho}:=C(\varrho) \omega_{\varrho}
$$

where

$$
C=C(\varrho):=\frac{1}{\left(k_{0} \bar{b} \tilde{\gamma}\right)^{\frac{1}{q-1}}\left(\bar{\eta}_{0} \varrho\right)^{\frac{q}{q-1}}}>0
$$

There exists $\varrho_{2} \in\left(0, \varrho_{1}\right]$ such that

$$
f_{\varrho}:=\left.g_{\varrho}\right|_{\bar{\jmath}}=\left.C(\varrho) \omega_{\varrho}\right|_{\bar{\jmath}}
$$

is a positive strict subsolution of (1) for each $\varrho \in\left(0, \varrho_{2}\right]$ and $\lambda \in\left(\delta^{\varrho}, \delta_{0}\right)$.

It follows that

$$
(-\Delta-\lambda) f_{\varrho}=C(\varrho)\left(\delta^{\varrho}-\lambda\right) \omega_{\varrho}<0
$$

for each $\varrho \in\left(0, \varrho_{1}\right]$ and $\lambda \in\left(\delta^{\varrho}, \delta_{0}\right)$.

It follows that

$$
\begin{aligned}
(\partial & +\chi(t)) f_{\varrho}(t)+\tilde{\gamma} \omega(t) f_{\varrho}^{q}(t) \\
& =C\left(\partial \omega_{\varrho}(t)+\chi(t) \omega_{\varrho}(t)+\tilde{\gamma} \omega(t) C^{q-1} \omega_{\varrho}^{q}(t)\right) \\
& \leq C\left(\partial \omega_{0}(t)+\varepsilon(1+\chi)+\tilde{\gamma} \bar{b} C^{q-1} \omega_{\varrho}^{q}\left(y_{\varrho}\right)\right) \\
& =C\left(-\eta_{0}(t)+\varepsilon(1+\chi)+\tilde{\gamma} \bar{b} C^{q-1}\left(\eta_{0}\left(z_{0}\right) \varrho+o(\varrho)\right)^{q}\right) \\
& \leq C\left(-\underline{\eta}_{0}+\varepsilon(1+\chi)+\tilde{\gamma} \bar{b} C^{q-1}\left(\bar{\eta}_{0} \varrho+o(\varrho)\right)^{q}\right) \\
& =C\left(-\underline{\eta}_{0}+\varepsilon(1+\chi)+\tilde{\gamma} \bar{b} C^{q-1}\left(\bar{\eta}_{0}^{q} \varrho^{q}+o\left(\varrho^{q}\right)\right)\right) \\
& =C\left(-\underline{\eta}_{0}+\varepsilon(1+\chi)+\frac{1}{k_{0}}+\frac{1}{k_{0} \bar{\eta}_{0}^{q}} \frac{o\left(\varrho^{q}\right)}{\varrho^{q}}\right)
\end{aligned}
$$

from (30), (31), (32), (38), (39), (40), (41) and (43), which, together with (34), shows that there exists $\varrho_{2} \in\left(0, \varrho_{1}\right]$ such that

$$
(\partial+\chi(t)) f_{\varrho}(t)+\tilde{\gamma} \omega(t) f_{\varrho}^{q}(t) \leq C\left(-\underline{\eta}_{0}+\varepsilon(1+\chi)+\frac{1}{k_{0}}+\frac{1}{k_{0} \bar{\eta}_{0}^{q}} \frac{o\left(\varrho^{q}\right)}{\varrho^{q}}\right)<0
$$

for each $\varrho \in\left(0, \varrho_{2}\right]$ and $\left.t \in\right\urcorner_{1}$.

And hence

$$
\partial f_{\varrho}+\chi(t) f_{\varrho}+\tilde{\gamma} \omega(t) f_{\varrho}^{q}<0
$$

where $t \in T_{1}$ and $\varrho \in\left(0, \varrho_{2}\right]$. 
So

$$
\left.\left.f_{\varrho} \mid\right\urcorner_{0}=C(\varrho) \omega_{\varrho} \mid\right\urcorner_{0}=0
$$

Combining (44), (45) and (46), we have

$$
\begin{aligned}
& \left.(-\Delta-\lambda) f_{\varrho}<0 \quad \text { in }\right], \\
& \left.f_{\varrho}=0 \quad \text { on }\right\urcorner_{0}, \\
& \left.\partial f_{\varrho}+\chi(t) f_{\varrho}+\tilde{\gamma} \omega(t) f_{\varrho}^{q}<0 \quad \text { on }\right\urcorner_{1}
\end{aligned}
$$

for $\varrho \in\left(0, \varrho_{2}\right]$ and $\lambda \in\left(\delta^{\varrho}, \delta_{0}\right)$.

It follows from (29) that there exists $\left.\tilde{y}_{\varrho} \in\right\urcorner_{1}$ satisfying

$$
\min _{t \in\urcorner_{1}} \omega_{\varrho}(t)=\omega_{\varrho}\left(\tilde{y}_{\varrho}\right)>0
$$

for each $\varrho \in\left(0, \varrho_{2}\right]$ and there exists $\left.\underline{z} \in\right\urcorner_{1}$ satisfying

$$
\omega_{\varrho}\left(\tilde{y}_{\varrho}\right)=\eta_{0}(\underline{z}) \varrho+o(\varrho)
$$

Combining (48) and (49), we obtain

$$
f_{\varrho}(t)=C \omega_{\varrho}(t) \geq C \omega_{\varrho}\left(\tilde{y}_{\varrho}\right)=\frac{\eta_{0}(z) \varrho+o(\varrho)}{\left(k_{0} \bar{b} \tilde{\gamma}\right)^{\frac{1}{q-1}}\left(\bar{\eta}_{0} \varrho\right)^{\frac{q}{q-1}}} \geq \frac{\underline{\eta}_{0} \varrho+o(\varrho)}{\left(k_{0} \bar{b} \tilde{\gamma}\right)^{\frac{1}{q-1}}\left(\bar{\eta}_{0} \varrho\right)^{\frac{q}{q-1}}}
$$

for each $t \in\urcorner_{1}$ and $\varrho \in\left(0, \varrho_{2}\right]$, which yields

$$
\begin{aligned}
\liminf _{\varrho \downarrow 0}(t) & \geq \lim _{\varrho \downarrow 0} \frac{\underline{\eta}_{0} \varrho+o(\varrho)}{k_{0}^{\frac{1}{q-1}} \tilde{\gamma}^{\frac{1}{q-1}} \bar{b}^{\frac{1}{q-1}} \bar{\eta}_{0}^{\frac{q}{q-1}} \varrho^{\frac{q}{q-1}}} \\
& =\lim _{\varrho \downarrow 0} \frac{\underline{\eta}_{0}}{k_{0}^{\frac{1}{q-1}} \tilde{\gamma}^{\frac{1}{q-1}} \bar{b}^{\frac{1}{q-1}} \bar{\eta}_{0}^{\frac{q}{q-1}} \varrho^{\frac{1}{q-1}}}=\infty
\end{aligned}
$$

uniformly on $\urcorner_{1}$.

Passing to the limit as $\delta \rightarrow 0$ in (26) and using (27)-(28), we have

$$
\int_{\beth} \chi\left(t_{1}\right)\left(\left(\omega_{1}-\omega_{2}\right)(\cdot, s)\right)_{\varepsilon}^{+} \zeta_{t_{2}} d t=0
$$

If we choose $t_{2} \zeta$ as a test function in (50), then we have

$$
\begin{aligned}
& \int_{\beth} \chi\left(t_{1}\right)\left(\left(\omega_{1}-\omega_{2}\right)(\cdot, s)\right)_{\varepsilon}^{+} \varsigma d t+\int_{\beth} \chi\left(t_{1}\right)\left(\left(\omega_{1}-\omega_{2}\right)(\cdot, s)\right)_{\varepsilon}^{+} t_{2} \zeta_{t_{2}} d t=0, \\
& \forall t \in[0, M], \forall \varsigma \in \mathrm{D}(\beth), \varsigma \geq 0, \quad d(\operatorname{supp}(\varsigma), \partial \beth)=\varepsilon_{0} .
\end{aligned}
$$


Define the following functions $\mathrm{h}$ and $\mathrm{g}$ in $\left(t_{1}^{1}, t_{1}^{2}\right)$ (resp. $\left.\left(t_{2}^{1}, t_{2}^{2}\right)\right)$ :

$$
\mathrm{h}\left(t_{1}\right)= \begin{cases}2\left(\frac{t_{1}-t_{1}^{1}}{\delta}\right)^{2} & \text { if } t_{1} \in\left[t_{1}^{1}, t_{1}^{1}+\frac{\delta}{2}\right] \\ 1-2\left(1-\frac{t_{1}-t_{1}^{1}}{\delta}\right)^{2} & \text { if } t_{1} \in\left(t_{1}^{1}+\frac{\delta}{2}, t_{1}^{1}+\delta\right] \\ 1 & \text { if } t_{1} \in\left(t_{1}^{1}+\delta, t_{1}^{2}-\delta\right] \\ 1-2\left(1-\frac{t_{1}^{2}-t_{1}}{\delta}\right)^{2} & \text { if } t_{1} \in\left(t_{1}^{2}-\delta, t_{1}^{2}-\frac{\delta}{2}\right] \\ 2\left(\frac{t_{1}^{2}-t_{1}}{\delta}\right)^{2} & \text { if } t_{1} \in\left(t_{1}^{2}-\frac{\delta}{2}, t_{1}^{2}\right]\end{cases}
$$

and

$$
\mathrm{g}\left(t_{2}\right)= \begin{cases}2\left(\frac{t_{2}-t_{2}^{1}}{\delta}\right)^{2} & \text { if } t_{2} \in\left[t_{2}^{1}, t_{2}^{1}+\frac{\delta}{2}\right], \\ 1-2\left(1-\frac{t_{2}-t_{2}^{1}}{\delta}\right)^{2} & \text { if } t_{2} \in\left(t_{2}^{1}+\frac{\delta}{2}, t_{2}^{1}+\delta\right], \\ 1 & \text { if } t_{2} \in\left(t_{2}^{1}+\delta, t_{2}^{2}-\delta\right], \\ 1-2\left(1-\frac{t_{2}^{2}-t_{2}}{\delta}\right)^{2} & \text { if } t_{2} \in\left(t_{2}^{2}-\delta, t_{2}^{2}-\frac{\delta}{2}\right], \\ 2\left(\frac{t_{2}^{2}-t_{2}}{\delta}\right)^{2} & \text { if } t_{2} \in\left(t_{2}^{2}-\frac{\delta}{2}, t_{2}^{2}\right],\end{cases}
$$

respectively (see [25]), where $t_{1}^{1}, t_{1}^{2} \in(0, W)$ and $t_{2}^{1}, t_{2}^{2} \in(0, w)$ satisfying that $t_{1}^{1}<t_{1}^{2}, t_{2}^{1}<t_{2}^{2}$,

$$
d\left(0, t_{1}^{1}\right)=d\left(0, t_{2}^{1}\right)=d\left(L, t_{1}^{2}\right)=d\left(l, t_{2}^{2}\right)=\varepsilon_{0}
$$

and $\delta$ is a positive real number.

We have

$$
\begin{aligned}
& \mathrm{h}\left(t_{1}^{1}\right)=\mathrm{h}\left(t_{1}^{2}\right)=\mathrm{g}\left(t_{2}^{1}\right)=\mathrm{g}\left(t_{2}^{2}\right)=0, \\
& \mathrm{~h} \in C^{2}\left(\left[t_{1}^{1}, t_{1}^{2}\right]\right), \quad \mathrm{g} \in C^{2}\left(\left[t_{2}^{1}, t_{2}^{2}\right]\right),
\end{aligned}
$$

and $h, g \geq 0$.

If we set

$$
\beth_{\varepsilon_{0}}=\left(t_{1}^{1}, t_{1}^{2}\right) \times\left(t_{2}^{1}, t_{2}^{2}\right)
$$

then we see that $\mathrm{hg}^{2} \in C^{2}\left(\overline{\bar{\beth}_{1,2}}\right)$ and $\mathrm{hg}^{2} \geq 0$.

Let us extend $\mathrm{hg}^{2}$ outside $\beth_{\varepsilon_{0}}$ by 0 and still denote by hg ${ }^{2}$ this function. We obtain

$$
\begin{aligned}
0= & \int_{\beth_{\varepsilon_{0}}} \chi\left(t_{1}\right)\left(\left(\omega_{1}-\omega_{2}\right)(\cdot, s)\right)_{\varepsilon}^{+} \mathrm{hg}^{2} d t \\
& +2 \int_{\beth_{\varepsilon_{0}}} \chi\left(t_{1}\right)\left(\left(\omega_{1}-\omega_{2}\right)(\cdot, s)\right)_{\varepsilon}^{+} t_{2} \operatorname{hgg}^{\prime} d t \\
:= & \mathcal{N}_{\delta}^{1}+\mathcal{N}_{\delta}^{2}
\end{aligned}
$$

by choosing $\varsigma=\mathrm{hg}^{2}$ as a test function in (51).

We have

$$
\lim _{\delta \rightarrow 0} \mathcal{N}_{\delta}^{1}=\int_{\beth_{\varepsilon_{0}}} \chi\left(t_{1}\right)\left(\left(\omega_{1}-\omega_{2}\right)(\cdot, s)\right)_{\varepsilon}^{+} d t
$$


and

$$
\begin{aligned}
\left|\mathcal{N}_{\delta}^{2}\right|= & 2 \mid \int_{t_{1}^{1}}^{t_{1}^{2}} \int_{t_{2}^{1}}^{t_{2}^{1}+\delta} \chi\left(t_{1}\right)\left(\left(\omega_{1}-\omega_{2}\right)(\cdot, s)\right)_{\varepsilon}^{+} t_{2} \mathrm{hgg}^{\prime} d t \\
& +\int_{t_{1}^{1}}^{t_{1}^{2}} \int_{t_{2}^{2}-\delta}^{t_{2}^{2}} \chi\left(t_{1}\right)\left(\left(\omega_{1}-\omega_{2}\right)(\cdot, s)\right)_{\varepsilon}^{+} t_{2} \mathrm{hgg}^{\prime} d t \mid \\
\leq & C\left\{\int_{t_{1}^{1}}^{t_{1}^{2}} \int_{t_{2}^{1}}^{t_{2}^{1}+\delta}\left(\left(\omega_{1}-\omega_{2}\right)(\cdot, s)\right)_{\varepsilon}^{+} g\left|g^{\prime}\right| d t\right. \\
& \left.+\int_{t_{1}^{1}}^{t_{1}^{2}} \int_{t_{2}^{2}-\delta}^{t_{2}^{2}}\left(\left(\omega_{1}-\omega_{2}\right)(\cdot, s)\right)_{\varepsilon}^{+} g\left|g^{\prime}\right| d t\right\} \\
:= & C\left(\mathcal{N}_{\delta}^{2,1}+\mathcal{N}_{\delta}^{2,2}\right),
\end{aligned}
$$

where

$$
C=\sup _{\left(t_{1}, t_{2}\right) \in \Xi}\left(\chi\left(t_{1}\right) t_{2} \mathrm{~h}\left(t_{1}\right)\right)
$$

Since the function

$$
t_{2} \mapsto\left(\int_{t_{1}^{1}}^{t_{1}^{2}}\left(\left(\omega_{1}-\omega_{2}\right)(\cdot, s)\right)_{\varepsilon}^{+} d t_{1}\right) g
$$

is right-continuous and vanishes at $t_{2}^{1}$, uniformly bounded independently of $\delta$ and $\left|g^{\prime}\right| \sim \frac{1}{\delta}$, we know that

$$
\lim _{\delta \rightarrow 0} \mathcal{N}_{\delta}^{2,1}=0
$$

Similarly

$$
\lim _{\delta \rightarrow 0} \mathcal{N}_{\delta}^{2,2}=0
$$

It follows from (55)-(56) that

$$
\lim _{\delta \rightarrow 0} \mathcal{N}_{\delta}^{2}=0
$$

by letting $\delta \rightarrow 0$ in (54).

Similarly, it follows from (53) and (57) that

$$
\int_{\beth_{\varepsilon_{0}}} \chi\left(t_{1}\right)\left(\left(\omega_{1}-\omega_{2}\right)(\cdot, s)\right)_{\varepsilon}^{+} d t=0
$$

by passing to the limit as $\delta \rightarrow 0$ in (52), where $t \in[0, M]$.

Finally, it follows that

$$
\int_{\beth_{\varepsilon_{0}}} \chi\left(t_{1}\right)\left(\left(\omega_{1}-\omega_{2}\right)(\cdot, s)\right)^{+} d t=0
$$

by letting $\varepsilon \rightarrow 0$ in (58), where $t \in[0, M]$. 
So

$$
\int_{\beth} \chi\left(t_{1}\right)\left(\left(\omega_{1}-\omega_{2}\right)(\cdot, s)\right)^{+} d t=0,
$$

where $t \in[0, M]$, which yields

$$
\chi\left(t_{1}\right)\left(\left(\omega_{1}-\omega_{2}\right)(\cdot, s)\right)^{+}=0 \quad \text { a.e. in J }
$$

for all $t \in[0, M]$.

Thanks to (2), we deduce that

$$
\left(\left(\omega_{1}-\omega_{2}\right)(\cdot, s)\right)^{+}=0 \quad \text { a.e. in I }
$$

for all $t \in[0, M]$ (see [32]).

So

$$
\omega_{1} \leq \omega_{2} \quad \text { a.e. in } P .
$$

By exchanging the roles of $\omega_{1}$ and $\omega_{2}$, we obtain

$$
\omega_{2} \leq \omega_{1} \quad \text { a.e. in } P .
$$

We conclude that

$$
\omega_{2}=\omega_{1} \quad \text { a.e. in } P .
$$

Hence, (14) holds. If we combine (13) and (14), we see that the solution of problem (1) associated with the initial data $\omega_{0}$ is unique.

\section{Examples}

In this section, two boundary value problems involving nonlocal integral boundary conditions will be tested by using the present method.

Example 4.1 Consider the boundary value problem

$$
\begin{aligned}
& \left({ }^{c} D^{5 / 2}+3^{c} D^{3 / 2}+2^{c} D^{1 / 2}\right) x(t)=\frac{A}{\sqrt{t^{2}+49}}\left(\cos x+\tan ^{-1} t\right), \quad 0<t<1, \\
& x(0)=0, \quad x(1 / 3)=0, \quad x(1)=\int_{0}^{1 / 5} x(s) d s .
\end{aligned}
$$

Here, $\delta=1 / 2, \sigma=3 / 5, \xi=1 / 3, p_{2}=1, p_{1}=3, p_{0}=2, \lambda=1, A$ is a positive constant and

$$
f(t, x)=\frac{A}{\sqrt{t^{2}+49}}\left(\cos x+\tan ^{-1} t\right)
$$

Clearly the constants $p_{2}, p_{1}$, and $p_{0}$ satisfy the condition of Lemma 2.1 , and

$$
|f(t, x)-f(t, y)| \leq A|x-y| / 7
$$


where $\ell=A / 7$. Using the given values, we find $\alpha \approx 0.44269$ and $\alpha_{1} \approx 0.21725$, It is easy to check that

$$
|f(t, x)| \leq \frac{A(2+\pi)}{2 \sqrt{t^{2}+49}}=\theta(t)
$$

and $\ell \alpha_{1}<1$ when $A<32.22094$. As all the conditions of Theorem 3.1 are satisfied the problem (59)-(60) has at least one solution on $[0,1]$. On the other hand, $\ell \alpha<1$ whenever $A<15.81242$ and thus there exists a unique solution for the problem (59)-(60) on $[0,1]$ by Theorem 3.1 .

Example 4.2 Consider the boundary value problem

$$
\begin{aligned}
& \left({ }^{c} D^{5 / 2}+3^{c} D^{3 / 2}+2^{c} D^{1 / 2}\right) x(t)=\frac{1}{4 \pi} \sin (2 \pi x)+\frac{|x|^{2}}{1+|x|^{2}}, \quad 0<t<1, \\
& x(0)=0, \quad x(1 / 3)=0, \quad x(1)=\int_{0}^{1 / 5} x(s) d s .
\end{aligned}
$$

Here, $\delta=1 / 2, \sigma=3 / 5, \xi=1 / 3, p_{1}^{2}-4 p_{2} p_{0}=1>0, \lambda=1$, and

$$
f(t, x)=\frac{1}{4 \pi} \sin (2 \pi x)+\frac{|x|^{2}}{1+|x|^{2}}
$$

Clearly

$$
|f(t, x)| \leq\left|\frac{1}{4 \pi} \sin (2 \pi x)+\frac{|x|^{2}}{1+|x|^{2}}\right| \leq \frac{1}{2}\|x\|+1
$$

where $g(t)=1, \psi(\|x\|)=\frac{1}{2}\|x\|+1$.

Then, by using the condition (A4), we find that $K>0.56853$ (we have used $\alpha=0.44269$ ). Thus, the conclusion of Theorem 3.1 applies to problem (61)-(62).

\section{Conclusion}

This paper was devoted to modifying the Schrödinger-type identity related to the singular boundary value problem. We also presented some mathematical consequences of the method including a stability result. The main technical tools used to develop the mathematical analysis are local and global bifurcation, monotonicity techniques, fixed point theory in $b$-metric spaces and the maximum principle approach with respect to the Schrödinger operator. As an application, the uniqueness of solutions for singular boundary value problem for the Schrödinger equation was proved.

Acknowledgements

Not applicable.

Funding

This work is supported by the National Natural Science Foundation of China (No. U1304610) and National Program on Key Basic Research Project (No. 2015CB856003). 
Availability of data and materials

Not applicable.

Ethics approval and consent to participate

Not applicable.

Competing interests

The authors declare that they have no competing interests.

Consent for publication

Not applicable.

\section{Authors' contributions}

The authors completed the paper and approved the final manuscript.

\section{Author details}

'Department of Basic Teaching, Zhengzhou University of Industrial Technology, Zhengzhou, China. ${ }^{2}$ School of Mathematics and Statistics, Henan University, Kaifeng, China.

\section{Publisher's Note}

Springer Nature remains neutral with regard to jurisdictional claims in published maps and institutional affiliations.

\section{Received: 12 April 2019 Accepted: 5 September 2019 Published online: 14 September 2019}

\section{References}

1. Zhang, X., Liu, D., Yan, Z., Zhao, G., Yuan, Y.: Schrödinger-type identity for Schrödinger free boundary problems. Bound. Value Probl. 2018, 135 (2018)

2. Liu, F., Chen, T., Wu, H.: A note on the endpoint regularity of the Hardy-Littlewood maximal functions. Bull. Aust. Math. Soc. 94(1), 121-130 (2016)

3. Fan, J., He, F., Ren, K.: Fixed point theorem with Jachymski function in dislocated quasi-metric spaces. Math. Appl. $31(1), 42-48(2018)$

4. Gilardi, G.: A new approach to evolution free boundary problems. Commun. Partial Differ. Equ. 4, 1099-1123 (1979)

5. Rossi, J.D.: The blow-up rate for a semilinear parabolic equation with a nonlinear boundary condition. Acta Math. Univ. Comen. 67, 343-350 (1998)

6. Dibenedetto, E., Friedman, A.: Periodic behaviour for the evolutionary dam problem and related free boundary problems. Commun. Partial Differ. Equ. 11, 1297-1377 (1986)

7. Polidoro, S., Ragusa, M.A.: Harnack inequality for hypoelliptic ultraparabolic equations with a singular lower order term. Rev. Mat. Iberoam. 24(3), 1011-1046 (2008)

8. Guariglia, E., Silvestrov, S.: Fractional-wavelet analysis of positive definite distributions and wavelets on $D^{\prime}(C)$. In: Silvestrov, S., Rančić, M. (eds.) Engineering Mathematics II, pp. 337-353. Springer, Berlin (2016)

9. Goubet, O., Hamraoui, E.: Blow-up of solutions to cubic nonlinear Schrödinger equations with defect: the radial case. Adv. Nonlinear Anal. 6(2), 183-197 (2017)

10. Colorado, E.: On the existence of bound and ground states for some coupled nonlinear Schrödinger-Korteweg-de Vries equations. Adv. Nonlinear Anal. 6(4), 407-426 (2017)

11. Khader, M.M., Adel, M.: Chebyshev wavelet procedure for solving FLDEs. Acta Appl. Math. 158(1), 1-10 (2018)

12. Rybalko, Y: Initial value problem for the time-dependent linear Schrödinger equation with a point singular potential by the unified transform method. Opusc. Math. 38(6), 883-898 (2018)

13. Zhang, G., Gu, T.: Stable solitary waves for a class of nonlinear Schrödinger system with quadratic interaction. Electron. J. Qual. Theory Differ. Equ. 2018, 97 (2018)

14. Scapellato, A.: Regularity of solutions to elliptic equations on Herz spaces with variable exponents. Bound. Value Probl. 2019, 2 (2019)

15. Meng, B.: Minimal thinness with respect to the Schrödinger operator and its applications on singular Schrödinger-type boundary value problems. Bound. Value Probl. 2019, 88 (2019)

16. Meng, B:: Schrödinger-type identity to the existence and uniqueness of a solution to the stationary Schrödinger equation. Bound. Value Probl. 2019, 91 (2019)

17. Yang, J., Ma, W., Qin, Z.: Lump and lump-soliton solutions to the (2+1)-dimensional Ito equation. Anal. Math. Phys. 1, $1-10(2017)$

18. Zhang, L., Meng, X.: Evolutionary dynamics in a Lotka-Volterra competition model with impulsive periodic disturbance. Math. Methods Appl. Sci. 39(2), 177-188 (2016)

19. Xu, X., Sun, Y.: Two symmetry constraints for a generalized Dirac integrable hierarchy. J. Math. Anal. Appl. 458 1073-1090 (2018)

20. Tian, Z., Tian, M., Gu, C., Hao, X.: An accelerated Jacobi-gradient based iterative algorithm for solving Sylvester matrix equations. Filomat 31(8), 2381-2390 (2017)

21. Cui, Y., Ma, W., Sun, Q., Su, X.: New uniqueness results for boundary value problem of fractional differential equation. Nonlinear Anal., Model. Control 23(1), 31-39 (2018)

22. Polidoro, S., Ragusa, M.A.: On Some Schrödinger Type Equations. More Progresses in Analysis. World Scientific, Singapore (2009)

23. Meng, B., Wang, X.: Adaptive synchronization for uncertain delayed fractional-order Hopfield neural networks via fractional-order sliding mode control. Math. Probl. Eng. 2018, 1603629 (2018)

24. Amann, H.: Nonlinear elliptic equations with nonlinear boundary conditions. In: Eckhaus, W. (ed.) New Developments in Differential Equations. Math Studies, vol. 21, pp. 43-63. North-Holland, Amsterdam (1976) 
25. Gilbarg, D., Trudinger, N.S.: Elliptic Partial Differential Equations of Second Order. Springer, New York (1983)

26. Stein, E.M.: Singular Integrals and Differentiability Properties of Functions. Princeton University Press, Princeton (1970)

27. Mozer, J.: A new proof of De Giorgi's theorem concerning the regularity problem for elliptic differential equations. Commun. Pure Appl. Math. 13(3), 457-468 (1960)

28. Miller, K.S., Ross, B.: An Introduction to the Fractional Calculus and Fractional Differential Equations. Wiley, New York (1993)

29. Serrin, J.: Local behavior of solutions of quasilinear elliptic equations. Acta Math. 111, 247-302 (1964)

30. Zhikov, V.V.: Averaging of functionals of the calculus of variations and elasticity theory. Izv. Akad. Nauk SSSR, Ser. Mat. 50(4), 675-711 (1986)

31. Acerbi, E., Fusco, N.: A transmission problem in the calculus of variations. Calc. Var. Partial Differ. Equ. 2, 1-16 (1994)

32. Rabinowitz, P.H.: Some global results for nonlinear eigenvalue problems. J. Funct. Anal. 7, 487-513 (1971)

\section{Submit your manuscript to a SpringerOpen ${ }^{\circ}$} journal and benefit from:

- Convenient online submission

- Rigorous peer review

- Open access: articles freely available online

- High visibility within the field

- Retaining the copyright to your article

Submit your next manuscript at $\gg$ springeropen.com 